\title{
Compressive Wideband Power Spectrum Analysis for Eeg Signals Using Fastica And Neural Network
}

\author{
${ }^{1}$ R.Selvi, ${ }^{2}$ S.T.Sadish Kumar, ${ }^{3}$ Dr. Kasthuri. N \\ ${ }^{1}$ Student of M.E. ${ }^{2}$ M.E.,(PhD),Associate Professor ${ }^{3}$ Professor, Department of ECE. Nandha Engineering \\ College, Erode-52.
}

\begin{abstract}
In several applications, such as wideband spectrum sensing for cognitive radio, only the power spectrum (a.k.a. the power spectral density) is of interest and there is no need to recover the original signal itself. In addition, high-rate analog-to-digital converters (ADCs) are too power hungry for direct wideband spectrum sensing. These two facts have motivated us to investigate compressive wideband power spectrum sensing, which consists of a compressive sampling procedure and a reconstruction method that is able to recover the unknown power spectrum of a wide-sense stationary signal from the obtained sub-Nyquist rate samples.

The task oriented brain activity analysis and classification is a prime issue in EEG signal processing. The similar attempt has been done here to estimate the brain activity on the basis of power spectrum analysis. For this, the modified approach involving both Independent Component Analysis (ICA) and Principal Component Analysis (PCA) methodologies has been used in this paper to investigate the behavior of brain's electrical activity for a simple case of visual attention.The input EEG signals are analyzed with the aid of Fast Independent Component Analysis (FastICA), a Statistical Signal Processing Technique, to obtain the components related to the detection of epileptic seizures. The BackPropagation Neural Network is trained with the obtained components for effective detection of epileptic seizures.
\end{abstract}

Index Terms : EEG signal, ICA and PCA, BPNN, ANN.

\section{ELECTROENCEPHALOGRAPHY (EEG) SIGNAL}

\section{Introduction}

Electroencephalography is a medical imaging technique that reads scalp electrical activity generated by brain structures. The electroencephalogram (EEG) is defined as electrical activity of an alternating type recorded from the scalp surface after being picked up by metal electrodes and conductive media. The EEG measured directly from the cortical surface is called electrocardiogram while when using depth probes it is called electrogram. Thus electroencephalographic reading is a completely non-invasive procedure that can be applied repeatedly to patients, normal adults, and children with virtually no risk or limitation. When brain cells are activated, local current flows are produced. The intervention of human brain signal. EEG measures mostly the currents that flow during synaptic excitations of the dendrites of many pyramidal neurons in the cerebral cortex.

The differences of electrical potentials are caused by summed postsynaptic graded potentials from pyramidal cells that create electrical dipoles between soma (body of neuron) and apical dendrites. Brain electrical current consists mostly of $\mathrm{Na}+, \mathrm{K}+, \mathrm{Ca}++$, and $\mathrm{Cl}$ - ions that are pumped through channels in neuron membranes in the direction governed by membrane potential the detailed microscopic picture is more sophisticated, including different types of synapses involving variety of neurotransmitters.

Evoked potentials or event-related potentials (ERPs) are significant voltage fluctuations resulting from evoked neural activity. Evoked potential is initiated by an external or internal stimulus . Mental operations, such as those involved in perception, selective attention, language processing and memory, proceed over time ranges in the order of tens of milliseconds. Whereas PET and MRI can localize regions of activation during a given mental task, ERPs can help in defining the time course of these activations amplitudes of ERP components are often much smaller than spontaneous EEG components, so they are not to be recognized from raw EEG trace. They are extracted from set of single recordings by digital averaging of epochs of EEG time-locked to repeated occurrences of sensory, cognitive, or motor events.

\section{Eeglab}

EEGLAB, runs under the cross-platform MATLAB environment for processing collections of singletrial and/or averaged EEG data of any number of channels. Available functions include EEG data, channel and event information importing, data visualization, preprocessing, independent component analysis (ICA) and time/frequency decompositions including channel and component cross-coherence supported by bootstrap statistical methods based on data resampling. 
The simple example with the real EEG data is considered in order to resolve the sources of the artifacts and the sources of useful signal. It is also emphasized the clinical significance of each component and hence the importance of ICA method in clinical practice. Autoregressive (AR) spectral estimation techniques are known to provide better resolution than classical periodogram methods when short segments of data are select for analysis. It has been observed that the energy in the EEG data segment is concentrated not in the beginning but somewhere in between the initial and the final positions thus confirming fact that EEG is a mixed delay signal. This position where the energy is concentrated has been obtained with the help of least squares wave shaping filter. It is also shown that the knowledge of the position where the energy in the signal is concentrated can be used in making a better spectral estimation of short segments of EEG data.

\section{Action Potentials}

\section{Methods}

The information transmitted by a nerve is called an action potential and are caused by an exchange of ions across the neuron membrane and action potential is a temporary change in the membrane potential that is transmitted along the axon. It is usually initiated in the cell body and normally travels in one direction. The membrane potential depolarizes, producing a spike. After the peak of the spike the membrane re-polarizes. The potential becomes more negative than the resting potential and then returns to normal. Action potential are initiated by many different types of stimuli; sensory nerves respond to many types of stimuli, such as chemical, light, electricity, pressure, touch and stretching. On the other hand, the nerves within the brain and spinal cord are mostly stimulated by chemical activity at synapses.

\section{Evoked Potentials}

Evoked potentials or event-related potentials (ERPs) are significant voltage fluctuations resulting from evoked neural activity. Evoked potential is initiated by an external or internal stimulus. ERPs are suitable methodology for studying the aspects of cognitive processes of both normal and abnormal nature. Mental operations, such as those involved in perception, selective attention, language processing, and memory, proceed over time ranges in the order of tens of milliseconds.

Amplitudes of ERP components are often much smaller than spontaneous EEG components, so they are not to be recognized from raw EEG trace. They are extracted from set of single recordings by digital averaging of epochs of EEG time-locked to repeated occurrences of sensory, cognitive, or motor events. The spontaneous background EEG fluctuations, which are random relatively to time point when the stimuli occurred, are averaged out, leaving the event-related brain potentials.

\section{The Proposed Eeg Signal Analysis}

EEG signals are the signatures of neural activities. They are captured by multiple-electrode EEG machines either from inside the brain, over the cortex under the skull, or certain locations over the scalp and can be recorded in different formats.

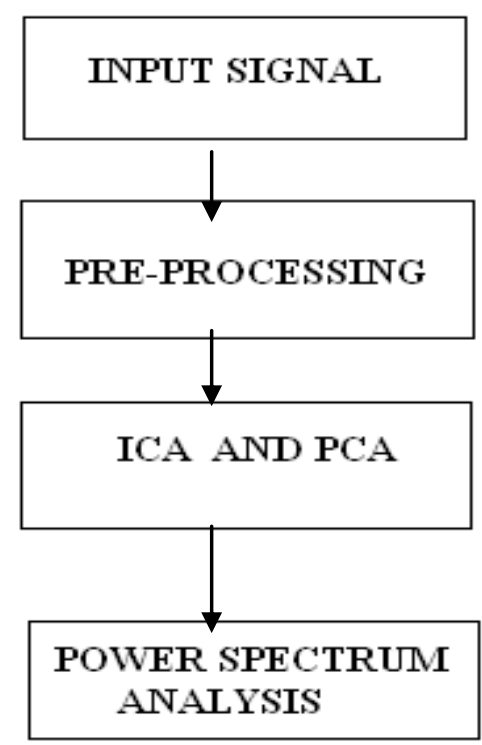

Figure 1. Flow graph of the process 
The figure 4.1 shows the flow graph of the process. The signals are normally presented in the time domain, but many new EEG machines are capable of applying simple signal processing tools such as the Fourier transform to perform frequency analysis and equipped with some imaging tools to visualize EEG topographies. There have been many algorithms developed so far for processing EEG signals. The operations include, but are not limited to, time-domain analysis, frequency-domain analysis, spatial-domain analysis, and multiway processing. Also, several algorithms have been developed to visualize the brain activity from images reconstructed from only the EEGs.

Spectral estimation helps in finding the pulse rhythms present in the EEG signal. The short segment of EEG data is analyzed for spectral parameters such as location and amount of spectral energy. The main objective of using prediction methods is to find a set of model parameters that best describe the signal generation system. Such models generally require a noise-type input. We need wavelet transform (WT) to analyze non-stationary signals, i.e., whose frequency response varies in time. Fourier transform (FT) is not suitable for non-stationary signals; wavelet transform decomposes a signal onto a set of basis functions called wavelets.

\subsection{Eeg Signal Pre-Processing}

The EEG signal filtering of recorded EEG signals has been done in the range of frequencies $0.1 \mathrm{~Hz}$ to $60 \mathrm{~Hz}$ by applying FIR filter of second order. At the same time, notch filter is also used to remove the power line interference in the channels spectrum around $50 \mathrm{~Hz}$. The artifacts are the disturbances in all or few channels due to eye blink or sometime by the low conductivity of electrodes. These disturbances are easily visible in time domain representation of the signals. These artifacts are present in all the channels, so that portion of data has been trimmed off manually from the signals all channels at once.

\subsection{Independent Component Analysis (Ica)}

The EEG signals are generally recorded through multi-channel lead system. Some important mutual information existing between pairs of channels is greatly valuable to reveal brain state information, build models, and establish locations etc. The most commonly used method for EEG processing are Fourier analysis and wavelet transform. These methods are appropriate for single channel EEG data because of its strong randomness and non-stationary nature. But these methods are not suitable for predicting the inter-dependence of the data of different channels in case of multichannel data processing. Most recently proposed the ICA algorithm is a statistical signal processing method, which can effectively separate the statistically independent source signals from observed multichannel signals, also used in improving the detection of changes in EEG . ICA can be simply described as follows: assuming that there are $\mathrm{N}$ number of observed signals $\mathrm{Xi}$, where $\mathrm{i}=1, \ldots, \mathrm{N}$, from $\mathrm{N}$ number of electrodes, and each signal is a linear mixture of $\mathrm{N}$ independent source signals $\mathrm{Si}$.

$$
X=A S
$$

Where $\mathrm{X}=[\mathrm{x} 1, \ldots, \mathrm{xN}] \mathrm{T}$ is observed signal vector and $\mathrm{S}=[\mathrm{s} 1, \ldots, \mathrm{SN}] \mathrm{T}$ is source signal vector, $\mathrm{A}$ is an unknown NxN mixing matrix. We expect that the source signal $\mathbf{S}$ can be separated from the observed signal vector $\mathbf{X}$. However, as the independent source $\mathbf{S}$ and mixing matrix $\mathbf{A}$ both are unknown, the traditional signal processing methods cannot solve the problem of blind source separation (BSS). Up to now, a lot of theories and algorithms for ICA have been presented and used to estimate the separation matrix $\mathbf{W}$ so that the independent sources can be successfully separated from observed multichannel signals, namely

$$
S=W X
$$

Independent Component Analysis (ICA) decomposes the input data using either logistic infomax ICA algorithm with the natural gradient feature or optionally the extended-ICA algorithm with sign estimation $\mathrm{N}$ training blocks. If $\mathrm{N}=0$, automatically estimate the number of sub-Gaussian sources, use single training block $(\mathrm{N}=1)$. The extended ICA has been followed here for input data decomposition.

\subsection{PRINCIPAL COMPONENT ANALYSIS (PCA)}

The PCA is a classical multivariate data analysis method that is useful in linear feature extraction, data compression and dimension reduction, sometimes used for back ground noise reduction . It is very much equivalent to Karhunen-LoCve transformation and closely related to factor analysis. These all methods follow the second order statistical computation. The PCA finds a linear transformation V $=\mathrm{Wu}$ such that the retained variance is maximized. It can be also viewed as a linear transformation which minimizes the reconstruction error. The row vectors of $\mathrm{W}$ correspond to the normalized orthogonal eigenvectors of the data covariance matrix.

Where $\mathrm{Uu}$ is the eigenvector matrix (i.e., modal matrix) and Du is the diagonal matrix whose diagonal elements correspond to the eigen values of Ru. Then the linear transformation $\mathrm{W}$ for PCA is dominant given by 
$\mathrm{W}=\mathrm{U}$ 'u .For dimensionality reduction, one can choose $\mathrm{p}$ column vectors in Uu that are eigenvectors that have the largest eigen dimensions. Annealing based on weight changes is used to automate the separation process. 32 components are now reduced to single value to construct a linear transform W. Conversion of all components into single component is done with PCA to reduce component.

\subsection{Fast Independent Component Analysis (Fast Ica)}

The epileptic seizures from the EEG brain signal are diagnosed with the aid of FastICA. The FastICA algorithm is an extremely efficient method for performing the estimation of ICA. The FastICA (Hyvärinen and Oja (1997); Hyvärinen (1999)) is one of the most well- known and popular algorithms for both independent component analysis (ICA) and blind source separation. For an m- element linear non- Gaussian signal mixture, the algorithm consists of a signal prewhitening stage followed by a set of $\mathrm{m}$ fixed-point iteration that extracts independent components using a non- Gaussianity signal measure. Coefficient vector orthogonality is used to guarantee uniqueness of the extracted components.The algorithm possess a number of valuable properties, including fast convergence, guaranteed global convergence for certain mixing conditions and contrasts.

Fast Independent Component Analysis (FastICA) algorithm separates the independent sources from their mixtures by measuring non-Gaussian. FastICA is a common offline method to identify artifact and interference from their mixtures such as Electroencephalogram (EEG), Magnetoencephalography (MEG), and Electrocardiogram (ECG). FastICA has been compared with neural-based adaptive algorithms and principal component analysis (PCA), and most ICA algorithms were found to outperform. Its popularity has been justified on the grounds of satisfactory performance offered by the method in several applications, as well as its simplicity .Other advantages of FastICA algorithm are: it can be used to perform projection pursuit and in addition it is used both in an exploratory fashion and also for estimating the independent components (or sources).

FastICA to the input signal (EEG), the proposed approach extracts the independent subcomponents corresponding to epileptic seizure from the mixture of EEG signals. This is followed by the training of the ascertained independent subcomponents, applying ANN (Artificial Neural Networks). Fig.2. depicts the block diagram of epileptic seizure detection process from EEG signal using FastICA and BackPropagation Neural Network (BPNN).

\subsection{Backpropagation Neural Network}

The epileptic seizure is separated from the EEG signals with the aid of Fast Independent Component Analysis, the training process will have to be carried out. Artificial Neural Networks (ANN) comes in handy for the training purposes and so it is utilized here. Literally speaking, the Artificial Neural Networks (ANN) is the elemental electronic delineation of the neural framework of the brain. An Artificial Neural Network is an adaptive, most often nonlinear system that learns to carry out a function (an input/output map) from data. The effect of the transformation is determined by the characteristics of the elements and the weights associated with the interconnections among them. By modifying theconnections between the nodes the network is able to adapt to the desired outputs.

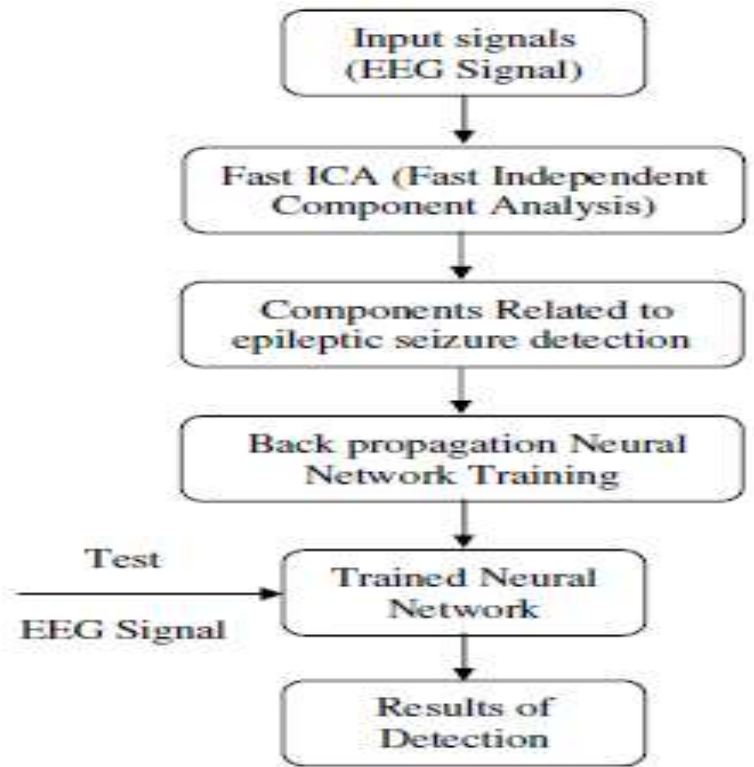

Fig 2. Block diagram of the proposed epileptic seizure detection approach 
The seizure affected parts of the brain can be identified once the Artificial Neural Networks are trained with the recorded EEG signals. In ANN, in fig.3. there are several techniques for training the input data. In the proposed approach, we use Back propagation algorithm for training the components obtained from the input (EEG) signals

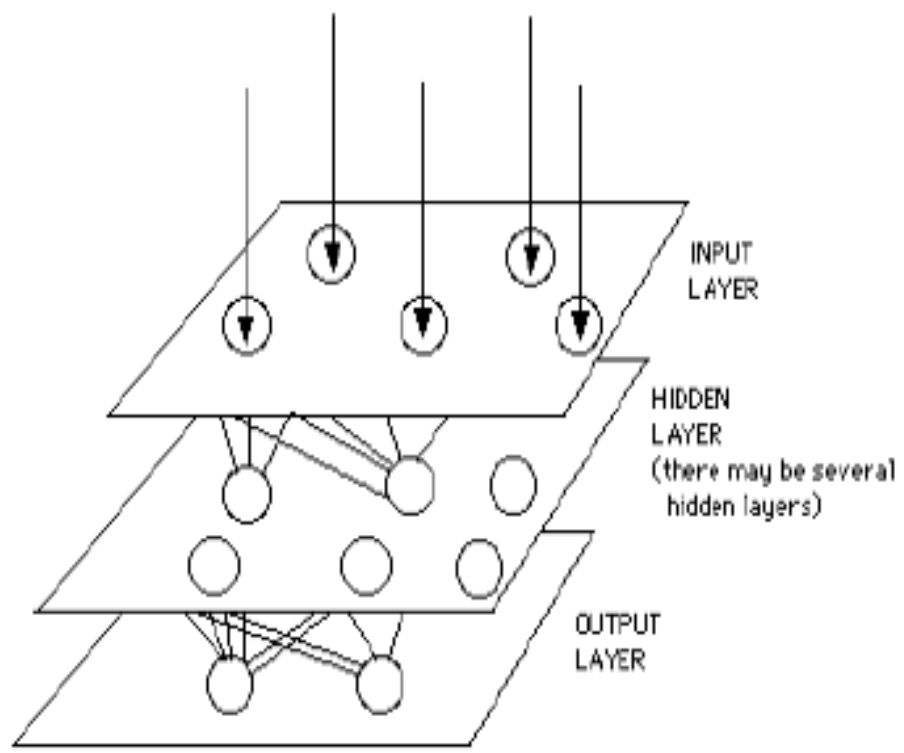

Fig 3. Architecture of Artificial Neural Networks

\subsection{Back Propagation Algorithm}

The EEG signals we utilize Back propagation algorithm. One of the most commonly used supervised ANN model is back propagation network that uses back propagation learning algorithm . In fig 4. shows the Back propagation algorithm is appropriate for pattern recognition problems. The back propagation neural network is essentially a network of simple processing elements working together to produce a complex output. These elements or nodes are arranged into different layers: input, middle and output . The advantages of Back propagation algorithm are, it is simple and its speed is also reasonable. The working procedure of back propagation algorithm.

Initially, a training input pattern is presented to the network input layer. The network propagates the input pattern from layer to layer until the output pattern is produced by the output layer. If this pattern is dissimilar from the preferred output, an error is intended and then propagated backward through the network from the output layer to the input layer. The weights are customized as the error is propagated.

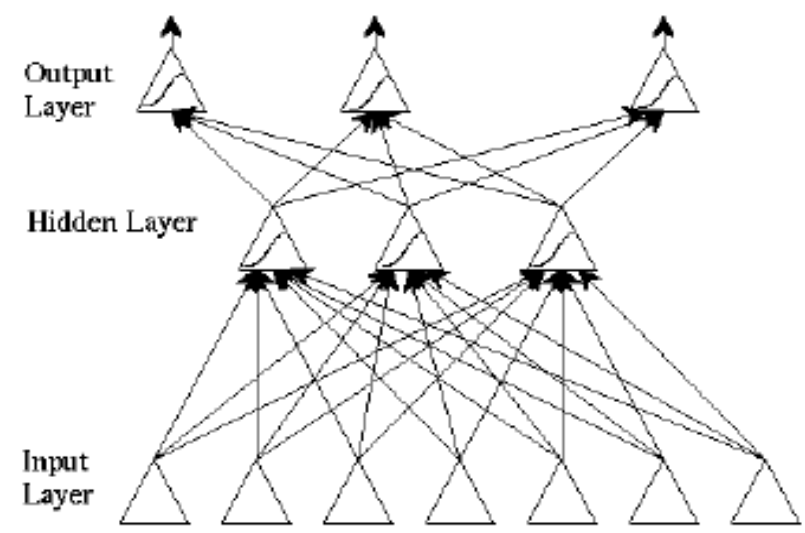

Fig 4. Back propagation neural network, the learning algorithm has two stages.

In the proposed approach, we primarily present the test input signal i.e. EEG signals to the neural network, which in turn compares the input signal with the trained signal, on the basis of the weight factor. If the input signals (EEG signal) contain epileptic seizure, then the trained network notifies the occurrence of the disease. The utilization of Back propagation shows better results in the detection of seizure from EEG signal. 


\section{Results}

The experimental results of the proposed approach for automatically detecting epileptic seizures in EEG signals using FastICA and back propagation neural network is detailed in this section. The presented approach is programmed in Matlab 7.8. The EEG signals experimented in the proposed approach was collected. The independent source $\mathbf{S}$ and mixing matrix $\mathbf{A}$ both are unknown, the traditional signal processing methods cannot solve the problem of blind source separation (BSS). Up to now, a lot of theories and algorithms for ICA have been presented and used to estimate the separation matrix $\mathbf{W}$ so that the independent sources can be successfully separated from observed multichannel signals.
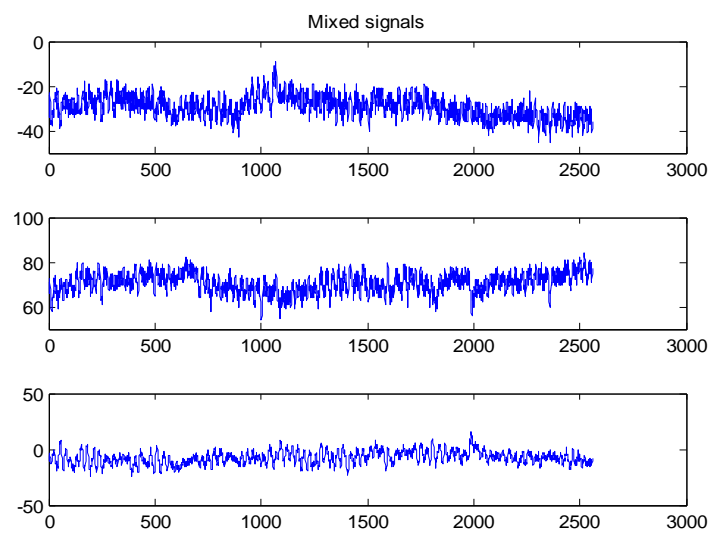

Fig 5.Mixed input signals for 6 channels.

Fig 5. shows that the matrix mixedsig contains the multidimensional signals as row vectors, and initialGuess gives the initial value for the mixing matrix used in the algorithm.FASTICA uses the fixed-point algorithm developed by Aapo Hyvarinen,
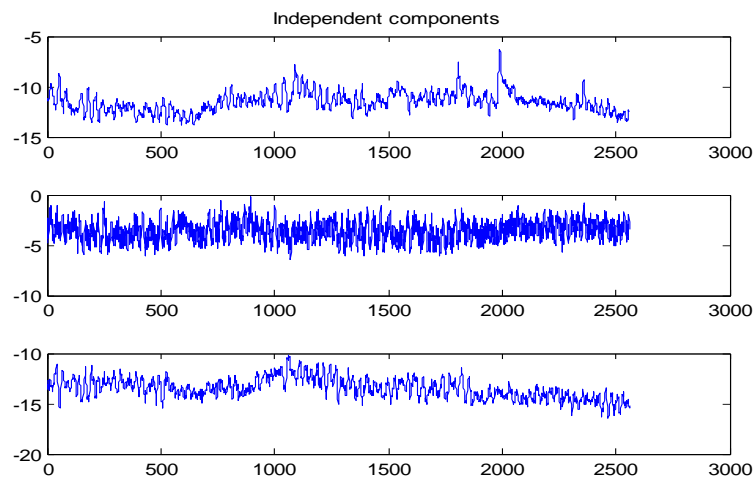

Fig 6.Independent component analysis

The fig 6 shows that the Independent Component Analysis plot is mainly for plotting and comparing the mixed signals and separated ica-signals. Independent component analysis plot has many different modes. The first parameter of the function defines the mode. Other parameters and their order depends on the mode. 


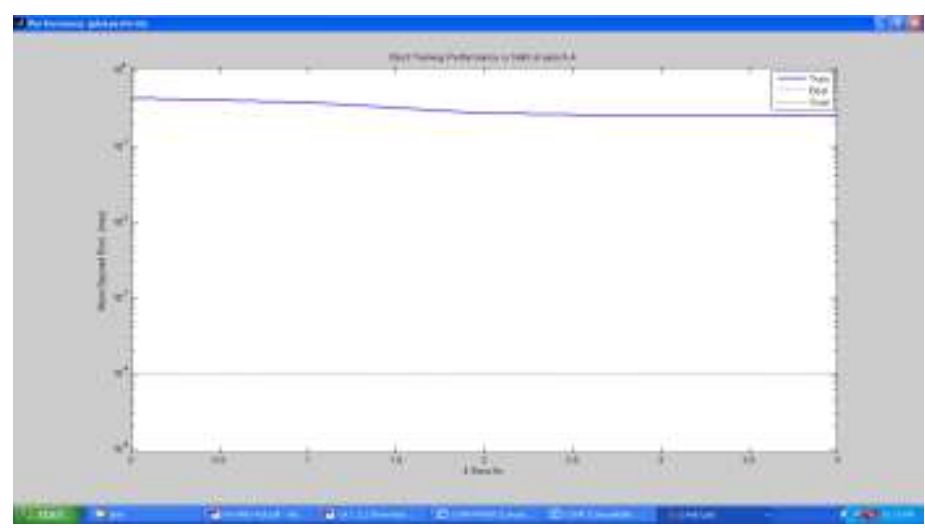

Fig 7.Performance plot

The training iteration is illustrated in Fig 7 using sets of 75 combinational data. For this case,fig 8.shows the training seemed to require 4 iterations in order to achieve the goal.

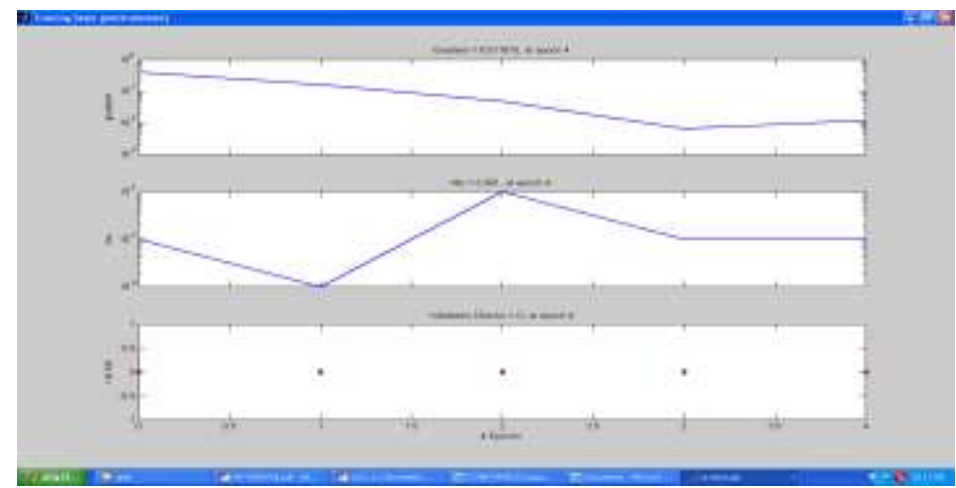

Fig 8.Training state

VI. Conclusion

Electroencephalography belongs to electro biological imaging tools which is widely used in medical and research areas. The advances signal processing techniques has been used for detection and separation of brain signals. The study proves that ICA is a powerful tool when the biomedical analysis involved more channels, which is the case of electroencephalogram. The important information can be obtained considering only the relevant signals, obtained after applying the Independent Component Analysis.The predominant purpose of this research is to achieve epileptic seizure detection from the recorded EEG brain signals. To begin with, independent subcomponents are separated from the recorded signals with the aid of Fast Independent Component Analysis. Further, the signals are trained using ANN (Artificial Neural Networks) technique namely Back propagation algorithm.

\section{References}

[1] Dyonisius Dony Ariananda, Student Member, IEEE, and Geert Leus, Fellow,IEEE (september 2012),IEEE transactions on signal processing, "Compressive Wideband Power Spectrum Estimation" Vol. 60, No.9,

[2] Mitul Kumar Ahirwal and Narendra D londhe, (March 2012) "Power Spectrum Analysis of EEG Signals for Estimating Visual Attention." Assistant Professors National Institute of Technology Raipur, Raipur-492010, International Journal of Computer Applications (0975 - 8887) Volume 42- No.15,

[3] Digital Analysis Of EEG Brain Signal Mr. Rash Dubey,(2011) Asst. Prof., E \& IE, APJ College of Engg., Sohna, Gurgaon, 121003 - India

[4] M. Mishali and Y. Eldar,( Apr. 2010) "From theory to practice: Sub-Nyquist sampling of sparse wideband analog signals," IEEE J. Sel. Top. Signal Process., Vol. 4, No. 2.

[5] Sivasankari. N and Dr. K. Thanushkodi Int. J. (2009) Automated Epileptic Seizure Detection in EEG Signals Using FastICA and Neural Network Advance. Soft Comput Appl., Vol. 1, No. 2,

[6] Lias, S., Sulaiman, N., Murat, Z.M., and Taib, M.N. 2010 IQ Index using Alpha-Beta Correlation of EEG Power Spectrum Density (PSD), Paper Presented at the IEEE Symposium on Industrial Electronics and Applications (ISIEA) Penang, Malaysia.

[7] EEG/ERP data vailable for free public download. Obtained through the Internet: http: //sccn.ucsd.edu / arno/fam2data/publicly_available_EEG_data.html, [accessed 15/01/2011].

[8] EEGLAB a Matlab tool box for EEG analysis Obtained through the Internet:http://sccn.ucsd.edu [accessed 12/11/2010].

[9] Hamid N.H.A., Sulaiman, N., Aris, S.M.A., Murat Z.H., and Taib, M.N., 2010 Evaluation of Human Stress Using EEG Power Spectrum, Paper Presented at the International Colloquium on Signal Processing \& Its Applications (CSPA).

[10] M. Kim and J. Takada, (Sep. 2009) "Efficient multichannel wideband spectrum sensing technique using filter bank," in Proc. IEEE Int. Symp. Pers. IndoorMobileRadio Commun. (PIMRC 2009), Tokyo, Japan, 